\title{
Dopamine and fear memory formation in the human amygdala
}

\author{
Andreas Frick (DD ${ }^{1 凶}$, Johannes Björkstrand ${ }^{2,3}$, Mark Lubberink ${ }^{4}$, Allison Eriksson $\mathbb{D}^{5}$, Mats Fredrikson ${ }^{3,6,8}$ and Fredrik Åhs $^{7,8}$
}

(c) The Author(s) 2021

Learning which environmental cues that predict danger is crucial for survival and accomplished through Pavlovian fear conditioning. In humans and rodents alike, fear conditioning is amygdala-dependent and rests on similar neurocircuitry. Rodent studies have implicated a causative role for dopamine in the amygdala during fear memory formation, but the role of dopamine in aversive learning in humans is unclear. Here, we show dopamine release in the amygdala and striatum during fear learning in humans. Using simultaneous positron emission tomography and functional magnetic resonance imaging, we demonstrate that the amount of dopamine release is linked to strength of conditioned fear responses and linearly coupled to learning-induced activity in the amygdala. Thus, like in rodents, formation of amygdala-dependent fear memories in humans seems to be facilitated by endogenous dopamine release, supporting an evolutionary conserved neurochemical mechanism for aversive memory formation.

Molecular Psychiatry (2022) 27:1704-1711; https://doi.org/10.1038/s41380-021-01400-x

\section{INTRODUCTION}

Fear conditioning is an evolutionary shaped mechanism for aversive memory formation important for survival. In fear conditioning, a previously neutral cue turns into a conditioned stimulus (CS) through pairings with an aversive stimulus [1] forming a memory trace in the amygdala [2-4], a key brain region supporting associative and emotional learning $[5,6]$. The amygdala is heavily innervated by dopamine [7], and in rodents, optogenetic stimulation of dopaminergic neurons [8], as well as systemic and amygdala-targeted administration of dopaminergic agonists, increase dopamine signaling and facilitate aversive learning [9]. In contrast, dopamine antagonists lead to attenuated memory formation [10]. Similarly, neurochemical lesions to the dopamine system in the amygdala severely compromise fear learning $[11,12]$, and fear conditioning does not occur in genetically dopamine-deficient mice, but is restored with administration of the dopamine precursor L-DOPA [13]. Dopamine release seems both necessary [13] and sufficient [14] for fear conditioning in rodents, suggesting causation and implicating that human fear learning is dopaminedependent. However, little is known of dopaminergic modulation of aversive learning in humans, but working memory $[15,16]$ and sequential learning [17] are facilitated by endogenous dopamine release in the striatum. In Parkinson patients with reduced dopamine function, amygdala-mediated fear processing is compromised but restored after dopamimetic treatment [18], and polymorphisms in genes encoding dopamine receptor 4 are associated with human fear conditioning [19]. However, no brain imaging study has directly evaluated if dopamine is released during amygdala-mediated associative learning or if the amount of dopamine released predicts learning strength.
In humans, positron emission tomography (PET) with the dopamine D2/D3 antagonist radiotracer $\left[{ }^{11} \mathrm{C}\right]$ raclopride has been widely used to assess endogenous dopamine release during psychological tasks and pharmacological challenges. In contrast to previous genetic and pharmacological studies showing the involvement of dopamine in fear learning in humans, but unable to localize dopaminergic signaling to certain brain regions $[18,19]$, $\left[{ }^{11} \mathrm{C}\right]$ raclopride PET provides spatial resolution of dopamine release and allows pinpointing specific brain regions involved. Although dopamine release in the amygdala is scarcely investigated in humans, it should be noted that protein expression of the dopamine 2 receptor is high in the human basolateral nucleus [20]. Further pointing to the feasibility of studying dopamine release in the amygdala, test-retest reliability of amygdala $\left[{ }^{11} \mathrm{C}\right]$ raclopride BP has been reported to be high [21].

To assess if fear memory formation in humans is dopaminerelated, we simultaneously measured brain dopamine release and neural activity in a combined positron emission tomography/ magnetic resonance imaging (PET/MRI) scanner during fear conditioning, with fear learning probed by skin conductance responses $(\mathrm{SCR})$ to a shock-predicting cue $(\mathrm{CS}+)$ and a control cue (CS-) never paired with shock (Fig. 1). We used single scan bolus/ infusion of $\left[{ }^{11} \mathrm{C}\right]$ raclopride $[21,22]$ to measure conditioning-related change in binding potential (i.e., dopamine release) [15], combined with functional magnetic resonance imaging (fMRI) to measure neural activity. We predicted that increased dopamine levels in the amygdala during fear conditioning would facilitate learning $[8,13,14]$, with greater dopamine release related to superior cue discrimination and enhanced amygdala-located fear engram formation. We also conducted exploratory analyses in the striatum, another brain region involved in fear conditioning rich in dopamine $D 2 / 3$ receptors [23].

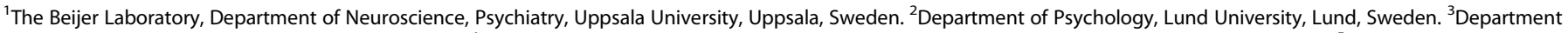

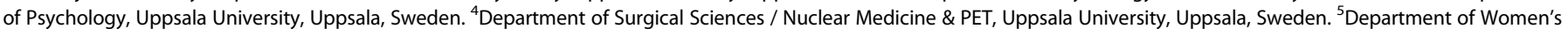

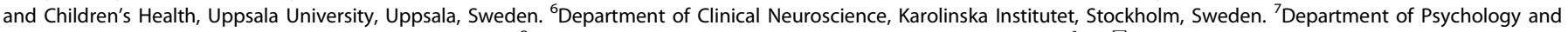
Social Work, Mid Sweden University, Östersund, Sweden. ${ }^{8}$ These authors contributed equally: Mats Fredrikson, Fredrik Åhs. ${ }^{\circledR}$ email: andreas.frick@neuro.uu.se
} 

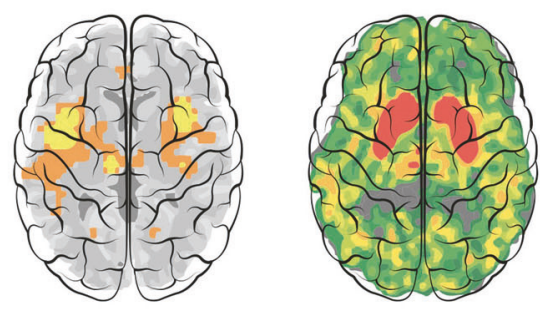

DOPAMINE

RELEASE

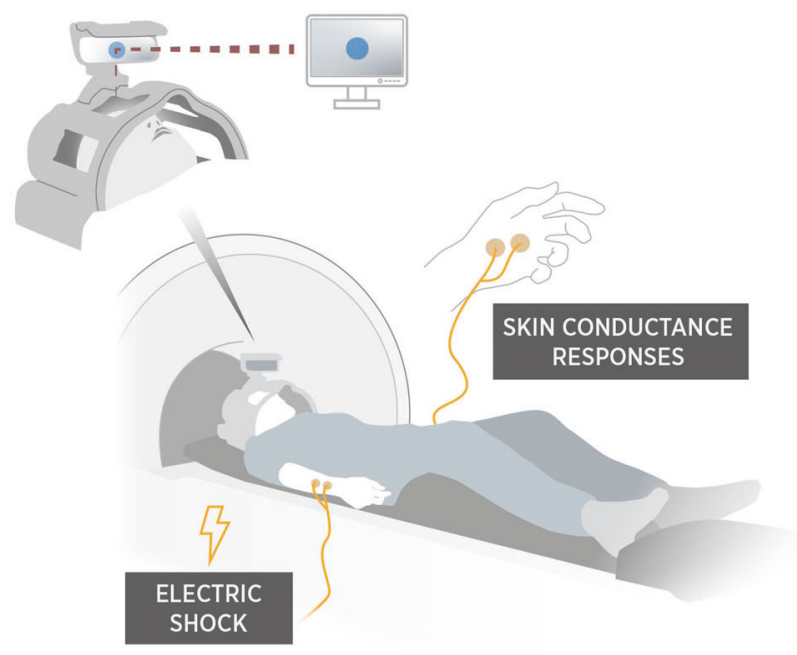

Fig. 1 Simultaneous measures of dopamine release and neural activity in the amygdala during fear conditioning probed with standard skin-conductance response. Positron emission tomography using single scan bolus plus constant infusion of $\left[{ }^{11} \mathrm{C}\right]$ raclopride was combined with event-related functional magnetic resonance imaging during fear conditioning, where one cue displayed on the screen was reinforced by a mild electric shock (CS+), while another control cue (CS-) never was paired with shock. Brain images for illustration only and do not depict the actual results of the study.

\section{MATERIALS AND METHODS}

\section{Experimental design}

Eighteen individuals (mean \pm SD age $25.2 \pm 4.8$ years; 10 women, 8 men; 16 right-handed, 2 left-handed) recruited through local advertisements were included in the study, which was approved by the regional ethics review board and radiation safety committee in Uppsala, Sweden. Participants arrived at the scanning site about $2 \mathrm{~h}$ before scanning. They were informed about the study and signed informed consent. Approximately $90 \mathrm{~min}$ prior to radiotracer injection and PET scanning, participants determined the strength of the unconditioned electric shock through a staircase procedure with the instruction that the shock should be unpleasant, but endurable.

Participants were positioned supine in the combined Signa 3T PET/MR scanner (GE Healthcare) with their heads lightly fixated inside the head coil. A bolus (20 s) of the selective dopamine D2/3 receptor antagonist $\left[{ }^{11} \mathrm{C}\right]$ raclopride was injected through a venous catheter and followed by constant infusion during the $90 \mathrm{~min}$ of PET data acquisition. Following 50 min of resting PET data collection, participants underwent a differential fear conditioning paradigm during collection of blood-oxygenation-level dependent (BOLD) fMRI and skin conductance. The PET scanning continued $20 \mathrm{~min}$ after the fear conditioning paradigm.

\section{Fear conditioning paradigm}

The fear conditioning paradigm lasted $20 \mathrm{~min}$ and consisted of 20 presentations each of two geometrical shapes (a brown arrow and a blue circle) used as conditioned stimuli (CS). One of the shapes (CS+) was paired with an electric shock on 16 occasions (80\% reinforcement rate), and the other (CS-) was unpaired [24]. The CSs were counterbalanced across subjects. Each CS was presented for $6 \mathrm{~s}$ with a mean $24.3 \mathrm{~s}$ fixation cross inter-trial interval varying between 21.8 and $27 \mathrm{~s}$. CS+ co-terminated with a 250 ms electric shock on reinforced trials.

Visual stimuli were projected onto a 32" computer screen positioned at the head of the scanner using E-prime 2 (Psychology Software Tools, Pittsburgh, PA, USA). Participants viewed the computer screen through a mirror on the head-coil. The presentation software was synced with fMRI data acquisition using a SyncBox (NordicNeuroLab, Bergen, Norway).

Electric shocks were used as unconditioned stimuli (US) and delivered to the subjects' dorsal right lower arm via disposable radiotransluscent electrodes (EL509, BIOPAC Systems, Goleta, CA, USA) by the STM100C module connected to the STM200 constant voltage stimulator and controlled by the BIOPAC MP150 (BIOPAC Systems, Goleta, CA, USA).

\section{Skin conductance responses}

Skin conductance was recorded using the BIOPAC MP150 (BIOPAC Systems, Goleta, CA, USA). Disposable radiotransluscent $\mathrm{Ag} / \mathrm{AgCl}$ electrodes (EL509 Biopac electrodes) were filled with isotonic electrode gel (GEL101 Biopac gel) and applied to the hypothenar eminence of the left hand. A $0.05 \mathrm{~Hz}$ high-pass filter was applied to the signal. SCRs were calculated as the maximum phasic driver amplitude 1-5 $\mathrm{s}$ after stimulus presentation using the Ledalab software [25] implemented in MATLAB (Mathworks Inc., Natick, MA) with responses $<0.01$ scored as 0 (nonresponse). SCRs were square root transformed and range-corrected by dividing each participant's SCRs with his/her maximum SCR, resulting in $\mathrm{SCR}$ ranging from 0 to 1 . This minimizes the influence of individual differences and isolates the experimental effects.

To evaluate fear conditioning acquisition, mean values of SCRs to CS+ and CS - were used to calculate delta scores (CS+ minus CS-) for each individual. Delta scores are independent of individual differences in general reactivity and habituation (response decline over successive trial presentations) as that affects $\mathrm{CS}+$ and $\mathrm{CS}-$ to an equal extent and thus control for nonspecific activity and represents an unbiased measure of associative learning with respect to general arousal. All methods are standard procedures and analytic strategies in fear conditioning [26, 27].

\section{PET/fMRI acquisition}

Subjects underwent a 90 min PET scan on a 3 T Signa PET-MR scanner (GE Healthcare, Waukesha), initiated simultaneously with the start of a $\left[^{11} \mathrm{C}\right]$ raclopride bolus-infusion protocol (total amount of radioactivity mean \pm SD $379 \pm 75 \mathrm{MBq}$; kbol $107 \mathrm{~min}$ ). Images were reconstructed into 185 -min frames using ordered subset expectation maximization (4 iterations, 28 subsets), including resolution recovery and a $5 \mathrm{~mm}$ Gaussian post-filter. Attenuation correction was done using the manufacturer's atlas-based method, and all other corrections necessary for quantitative PET images were applied. Atlas-based attenuation correction has been shown to be less accurate than CT-based or zero echo time (ZTE) MRI-based attenuation correction, but because we were only addressing changes in receptor binding within the same patient and scan, this does not affect our results. Head movement in the scanner was restricted using foam cushions.

Anatomical 3D T1-weighted images were acquired with an 8 channel head coil and the following parameters (repetition time $(T R)=8.6 \mathrm{~ms}$, echo time $(T E)=3.3 \mathrm{~ms}$, inversion time $=450 \mathrm{~ms}$, flip angle $=12$, matrix $=256 \times 256$, voxel size $=1.2 \times 1.2 \times 1.2 \mathrm{~mm}$ ), starting $\sim 15 \mathrm{~min}$ after bolus injection. BOLD fMRI was collected using a single shot echo-planar imaging (EPI) sequence with parameters $\left(\mathrm{TR}=3000 \mathrm{~ms}, \mathrm{TE}=30 \mathrm{~ms}\right.$, flip angle $90^{\circ}$, matrix $=64 \times 64$, voxel size $=3.0 \times 3.0 \mathrm{~mm}$, slice gap $=0.4 \mathrm{~mm}$, slices $=45$ ).

\section{PET analysis}

PET images were corrected for inter-frame motion using frame-by-frame alignment with Voiager software (GE Healthcare, Uppsala, Sweden). For each participant, the T1-weighted MRI image was co-registered to a summed PET image and segmented into gray matter, white matter and CSF using SPM8 (fil.ion.ucl.ac.uk/spm). A probabilistic volume of interest (VOI) template containing 45 VOls was applied to the co-registered T1weighted MRI image and transferred to the dynamic PET data using PVElab [28], resulting in gray matter time-activity curves (TAC) of the regions of interest.

PET data were analyzed both in VOls and voxel-by-voxel using a nested two-step approach: first, the initial 50 min of the TACs were analyzed using the simplified reference tissue model (SRTM) [29] for VOls and for voxelwise analyses a basis function implementation of SRTM [30] with cerebellar 

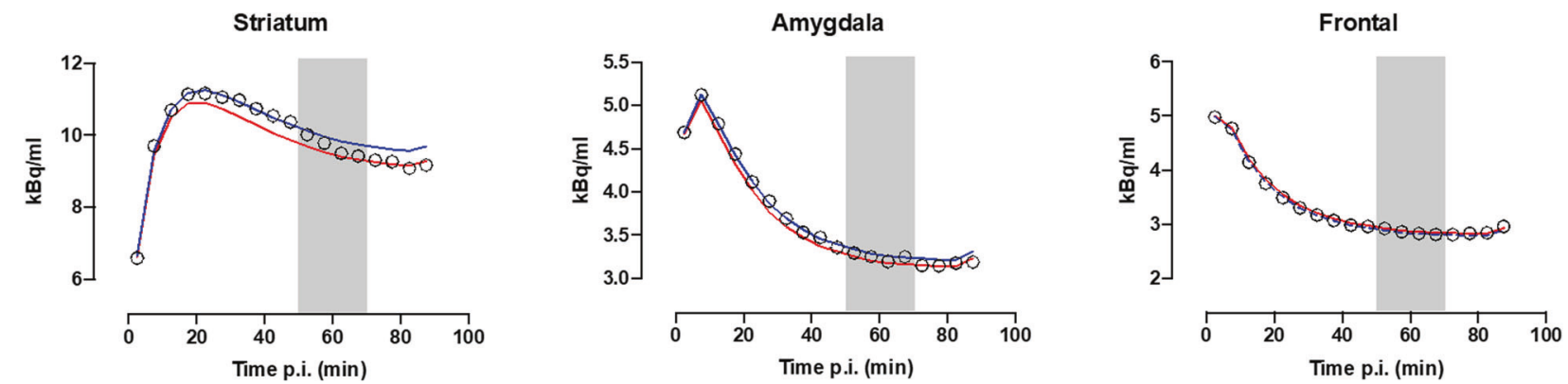

Fig. 2 Simplified reference tissue model fits. Mean time-activity curves in the striatum, amygdala, and frontal cortex. Time post injection (p.i.) minutes. Blue lines are fit lines of the simplified reference tissue model (SRTM) fits to the $0-50$ min baseline portion of the data, whereas red lines are fits to the $70-90$ min post conditioning portion of the data re-using all parameters from the baseline fit except $B P_{N D}$. The gray square denotes the timing of the fear conditioning at 50-70 min p.i. If there would be no dopamine release, i.e., no change in [ $\left.{ }^{11} \mathrm{C}\right] \mathrm{raclop}$ ride binding potential $\left(\mathrm{BP}_{\mathrm{ND}}\right)$, the time-activity data would follow the fit line of the $0-50 \mathrm{~min}$ data. As can be seen, the PET time-activity data in the amygdala and striatum no longer follow the expected (blue) curve from the start of fear conditioning at 50 min post injection, indicating dopamine release resulting in a reduced $\mathrm{BP}_{\mathrm{ND}}$. The fits to the mean time-activity curves correspond to a reduction in $\mathrm{BP}_{\mathrm{ND}}$ after challenge of $5.8 \%, 12.5 \%$, and $0 \%$ in the striatum, amygdala, and frontal cortex, respectively.

gray matter as reference tissue, giving baseline $R_{1}$ (tracer delivery relative to cerebellum), $\mathrm{k}_{2}^{\prime}$ (reference tissue efflux rate constant) and $\mathrm{BP}_{\mathrm{ND}}$ (nondisplaceable binding potential). Then, the same models were applied to the 70-90 min interval, fixing $R_{1}$ and $k_{2}^{\prime}$ at baseline values and only fitting for $\mathrm{BP}_{\mathrm{ND}}^{\prime}$, the binding potential after conditioning. The percent difference in binding potential was calculated as $\left[100 \times\left(1-\mathrm{BP}_{\mathrm{ND}}^{\prime} / \mathrm{BP}_{\mathrm{ND}}\right)\right]$ and used as a measure of endogenous dopamine release. The analyses resulted in VOI values and parametric images of $\mathrm{BP}_{\mathrm{ND}}, \mathrm{BP}_{\mathrm{ND}}^{\prime}$ and dopamine release. The parametric images were normalized to Montreal Neurological Institute (MNI) standard space in SPM12 by first co-registering each individual's parametric images to their T1-weighted MRI image, then segmenting and normalizing the T1-weighted image to $\mathrm{MNI}$ space, and finally applying the transformation parameters to the PET images. Images were resliced to 4 $\mathrm{mm}$ isotropic voxels and smoothed with an $8 \mathrm{~mm}$ full width half maximum (FWHM) Gaussian kernel.

An illustration of the fit of the two SRTM models to mean TACs is presented in Fig. 2. If there would be no dopamine release (i.e., no change in $\left[{ }^{11} \mathrm{C}\right]$ raclopride $\left.\mathrm{BP} \mathrm{ND}_{\mathrm{ND}}\right)$, the time-activity data would follow the fit line of the $0-50$ min data. As can be seen in Fig. 2, the time-activity data in the amygdala and striatum no longer follow the expected curve from the start of fear conditioning at $50 \mathrm{~min}$ post injection, indicating dopamine release resulting in a reduced $B P_{N D}$. This deviation cannot be seen in the frontal cortex, thus not indicating dopamine release in this region.

Ideally, the use of a bolus-infusion protocol results in a steady state, which would allow for use of simple radioactivity concentration ratios to measure occupancy instead of tracer kinetic modeling. However, the nested two-step SRTM method can account for deviations from steady state, which are nearly always present, whilst ratio methods cannot. Another, previously published analysis method (Ip-ntPET) [31] would have given us the time course of the dopamine release in addition to the magnitude. This method, requiring fitting of 6 parameters instead of the 4 parameters in the present work, is less robust because of the larger number of parameters. Since neurotransmitter release due to fear conditioning is likely of lower levels than that due to pharmacological challenges, robustness of the model to measure small changes in BP is necessary.

Robustness of measuring post-challenge $\mathrm{BP}_{\mathrm{ND}}$ was assessed by performing a simulation study. For dopamine release levels resulting in a reduction in $\mathrm{BP}_{\mathrm{ND}}$ of $0,5,10,15$ and $20 \%, 100$ time-activity curves were numerically simulated using published values of rate constants, typical noise levels, and baseline BP $\mathrm{ND}_{\mathrm{ND}}$ values of 2.6 (representing striatum), 0.3 (representing amygdala), and 0.07 (representing frontal cortex). Changes in $\mathrm{BP}_{\mathrm{ND}}$ during the scan were simulated by a linear reduction in $\mathrm{k}_{3}$ between 50 and $70 \mathrm{~min}$ post injection, and resulting time-activity curves were analyzed using the nested version of SRTM. In addition, 100 TACs with a range of $\mathrm{BP}_{\mathrm{ND}}$ changes between 0 and $30 \%$ were simulated for each of the baseline $\mathrm{BP}_{\mathrm{ND}}$ values. A minor bias in post fear conditioning $\mathrm{BP}_{\mathrm{ND}}$ of $0-2 \%$ and coefficient of variation (COV) of around $10 \%$ was found for baseline $\mathrm{BP}_{\mathrm{ND}}$ values of 0.3 (amygdala). For high baseline $\mathrm{BP}_{\mathrm{ND}}$ (striatum), there was a positive bias in post-challenge $\mathrm{BP}_{\mathrm{ND}}$ that was proportional to dopamine release levels, and a COV of around $2.5 \%$. For lower baseline BP ND $_{\text {(frontal }}$ cortex), a varying bias of $\pm 5 \%$ was seen with COV exceeding $40 \%$. Hence, for striatum, the nested SRTM method seems to result in a proportional underestimation of dopamine release, whereas this effect is much smaller for amygdala (Supplementary Figs. 1 and 2). Post-challenge $\mathrm{BP}_{\mathrm{ND}}$ for frontal cortex cannot be estimated reliably because of poor precision. Use of SUVR or Ip-ntPET resulted in considerably larger bias and variability (data not shown).

Thus, simulations show a high accuracy and precision of the two-step nested method applied in the present work provided that the dopamine release time course, modeled as a gamma variate function, peaks before 70 $\mathrm{min}$ and has a relatively slow reduction after that. These conditions were confirmed both by microdialysis experiments in rodents [32] and by the observation that we see a continually reduced signal after $70 \mathrm{~min}$ in our data, with no reversal to initial activity concentration values within the time course of the scans, suggesting a persisting change in dopamine levels. Furthermore, simulations showed that the occupancy values estimated by this model were insensitive to conditioning-induced changes in $R_{1}$.

\section{fMRI analysis}

FMRI data was preprocessed and modeled using SPM12 (fil.ion.ucl.ac.uk/ spm/software/spm12) implemented in MATLAB R2018a (Mathworks Inc., Natick, MA). Each individual's fMRI data was first slice-timing corrected, realigned and co-registered to the anatomical T1-weighted image, and then normalized to isotropic $4 \mathrm{~mm}$ voxels in the MNI standard space by applying the transformation parameters from the segmentation of the T1weighted image. Finally, the images were smoothed with an $8 \mathrm{~mm}$ FWHM Gaussian filter.

The first level model for each participant was fitted with onsets and durations of $\mathrm{CS}_{+}, \mathrm{CS}_{-}$, and shock (modeled as a stick function) convolved with the canonical haemodynamic response function from SPM12, together with the six realignment parameters from the realignment step, as regressors. Contrast images were created for CS +, CS - and US vs. baseline, and for the learning-related CS+ minus CS-. Mean BOLD responses within the regions of interest amygdala and striatum were extracted using the Wake Forest University School of Medicine (WFU) PickAtlas automatic anatomical labeling (AAL) definitions of these regions [33].

To address potential influence of motion on the results, in addition to including the realignment parameters in the first level model, we set a cutoff to keep subjects with head motion not exceeding one acquisition voxel (i.e., $3 \mathrm{~mm}$ ) in any direction [34]. We also calculated the total net movement during the fear conditioning task (mean \pm SD: $1.3 \pm 0.7 \mathrm{~mm}$ ) and examined the relation between this measure and change in $\left[{ }^{11} \mathrm{C}\right]$ raclopride $\mathrm{BP}_{\mathrm{ND}}$, but could not detect any association in the amygdala $(r=-0.01, p=0.960,95 \% \mathrm{Cl}:-0.48$ to 0.46$)$ or in the striatum VOls $(r=$ $0.24, p=0.343,95 \% \mathrm{Cl}:-0.26$ to 0.63$)$.

\section{Statistical analyses}

We applied three a priori regions of interest in our analyses, the amygdala, striatum, and frontal cortex (superior frontal gyrus). The amygdala was our primary target based on our hypotheses, the striatum (combined caudate nucleus, putamen and nucleus accumbens) was included based on the involvement in fear conditioning and rich dopamine receptor D2/3 
A

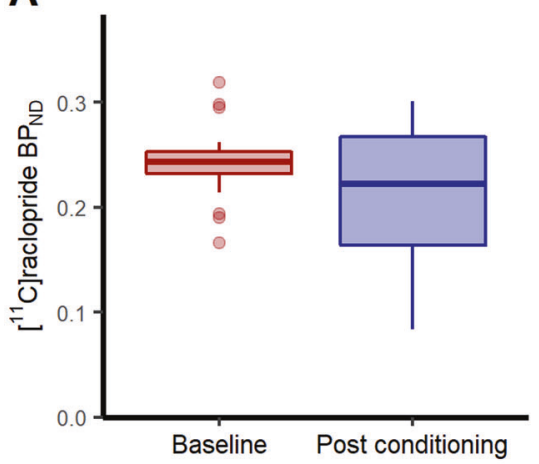

Amygdala

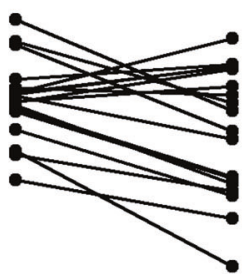

Baseline Post conditioning
B

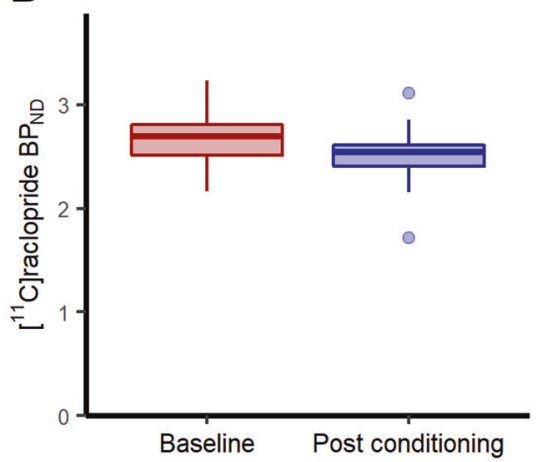

Striatum

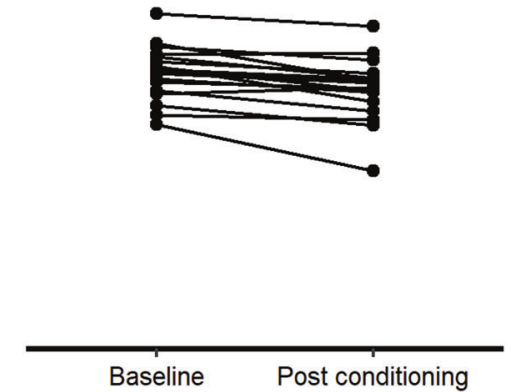

Fig. 3 Binding potential of $\left[{ }^{11} \mathrm{C}\right]$ raclopride at baseline and after fear conditioning. Boxplots and individual participant's trajectory lines showing $\left[{ }^{11} \mathrm{C}\right]$ raclopride binding potential $\left(\mathrm{BP}_{\mathrm{ND}}\right)$ at baseline and after fear conditioning in anatomically defined regions of interest. $\mathbf{A}$ In accordance with our hypothesis, $\left[{ }^{11} \mathrm{C}\right]$ raclopride $\mathrm{BP}_{\mathrm{ND}}$ in the amygdala decreased by $13.4 \%(95 \% \mathrm{Cl}: 3.8-22.9 \%)(t(17)=2.74, P=0.007)$ from baseline to after fear conditioning, indicating dopamine release. B Likewise, in the striatum, there was a $5.9 \%(95 \% \mathrm{Cl}$ : 3.4-8.4\%) decrease in $\mathrm{BP}_{\mathrm{ND}}(t(17)=4.69, P=0.0002)$. For the boxplots, the line indicates the median, the box the interquartile range (IQR), the whiskers the minimum of $1.5 \times \mathrm{IQR}$ and minimum/maximum values, and circles values more extreme than $1.5 \times \mathrm{IQR}$. Data for individual participants is shown in the trajectory lineplots.

expression in this region [23], and the frontal cortex (superior frontal gyrus), low in D2/3 receptor expression was our control region where we expected not to be able to measure dopamine release robustly.

Our main analyses involved the $\left[{ }^{11} \mathrm{C}\right]$ raclopride $\mathrm{BP}_{\mathrm{ND}}$ VOI values and mean BOLD responses within the amygdala and striatum. We also applied voxel-wise paired t-tests (baseline vs post-conditioning) in SPM12 to locate overlapping voxels with both lower $\left[{ }^{11} \mathrm{C}\right]$ raclopride $\mathrm{BP}_{\mathrm{ND}}$ (i.e., dopamine release) and learning-related neural activity (CS + minus CS- BOLD responses) from fear conditioning. The statistical threshold was set to $P<$ 0.05 family-wise error (FWE) corrected for multiple comparisons using random field theory and small volume correction within each region of interest (amygdala and striatum).

To assess the relations between dopamine release and central and peripheral learning, and between central and peripheral learning, we entered dopamine release and neural activity from the amygdala and striatum ROls into Pearson's product-moment correlations together with the learning-related delta SCR (CS+ minus CS-) in R version 4.0.0 [35]. We used directed tests $(P<0.05)$ due to the a priori hypotheses of positive correlations between these measures. Outlier detection testing Mahalanobis distance $(M D)$ against a $X^{2}$ distribution with 2 degrees of freedom and the standard criterion of $P<0.001$ revealed no bivariate outliers between PET, fMRI and SCR measures (amygdala: MDs <6.1, Ps >0.046; striatum: MDs <10.0, Ps $>0.007$ ).

\section{RESULTS}

\section{Dopamine release during fear conditioning}

First, using single scan bolus/infusion $\left[{ }^{11} \mathrm{C}\right]$ raclopride PET we found decreased binding potential $\left(\mathrm{BP}_{\mathrm{ND}}\right)$ from baseline to post conditioning in the amygdala (13.4\% reduction, 95\% Cl: 3.8-22.9\%, $t(17)=$ 2.74, $P=0.007$ ) (Fig. $3 \mathrm{~A})$, confirming the primary hypothesis of dopamine release in this region during fear conditioning. Additionally, exploratory analyses revealed decreased $\mathrm{BP}_{\mathrm{ND}}$ following conditioning in the striatum $(5.9 \%$ reduction, $95 \% \mathrm{Cl}: 3.4-8.4 \%$, $t(17)=4.69, P=0.0002$ ) (Fig. 3B), indicating dopamine release also in this brain area. In the frontal cortex, included here as a control region where we expected no decrease in $\mathrm{BP}_{\mathrm{ND}}$, we could not detect any change in $\mathrm{BP}_{\mathrm{ND}}$ between baseline and post fear conditioning (mean change: $-34.6 \%, 95 \% \mathrm{Cl}:-159.8 \%$ to $90.5 \%, t$ $(17)=0.54, P=0.595$ ) (Supplementary Fig. 3). Complementing these analyses of mean dopamine release in each region, we performed within region voxel-wise analyses, revealing reduced $\mathrm{BP}_{\mathrm{ND}}$ in bilateral amygdala clusters (left: $Z=3.22, P_{\mathrm{FWE}}=0.017$, $320 \mathrm{~mm}^{3}$, MNI peak voxel: $-22,0,-14$; right: $Z=3.35, P_{\mathrm{FWE}}=.012$, $960 \mathrm{~mm}^{3}$, MNI: 26, 0, -14) (Fig. 4) and in the striatum (left: $Z=6.24$; $P_{\mathrm{FWE}}<0.001,7488 \mathrm{~mm}^{3}$, MNI: $-18,8,-2$; right: $Z=6.54, P_{\mathrm{FWE}}<$ $0.001,8320 \mathrm{~mm}^{3}$, MNI: 22, 8, -2) (Fig. 5).

\section{Fear conditioning procedure induced learning}

Second, fear conditioning resulted in skin conductance response discrimination between fear and safety-predicting cues (CS+> $\mathrm{CS}-$ ), i.e., a peripheral expression of learning, as evidenced by the repeated measures ANOVA revealing main effects of CS $(F(1,17)=21.53, P=0.0002)$ and Trial $(F(19,323)=23.96, P<$ $0.00001)$, and a CSX Trial interaction $(F(19,323)=2.25, P=0.0023)$ (Fig. 4B). Simple main effects analyses revealed an effect of Trial for both $C S+(F(1,19)=16.79, P<0.001)$ and $C S-(F(1,19)=13.77$, $P<0.001)$, as well as an effect of CS for most trials, but importantly not for the first trial (i.e., before associative learning could have occurred) (Supplementary Table 1). These results and visual 
A

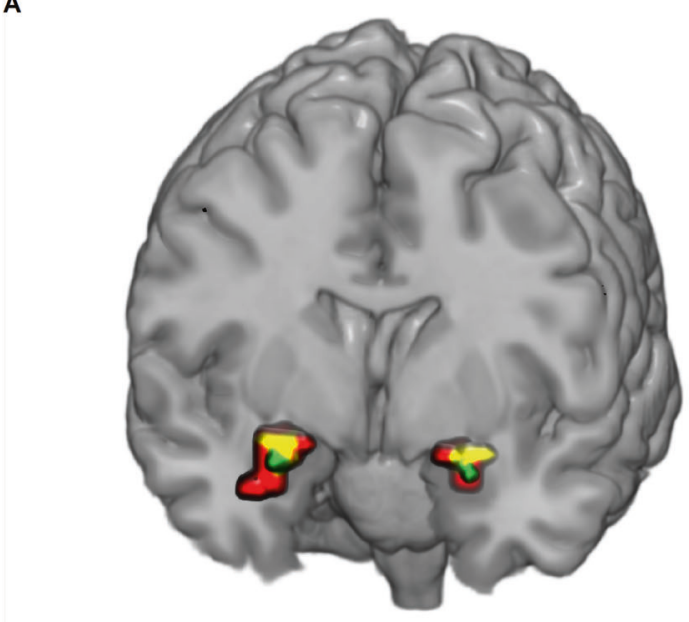

Dopamine release

$\mathrm{BOLD}(\mathrm{CS}+>\mathrm{CS}-)$

Dopamine BOLD overlap
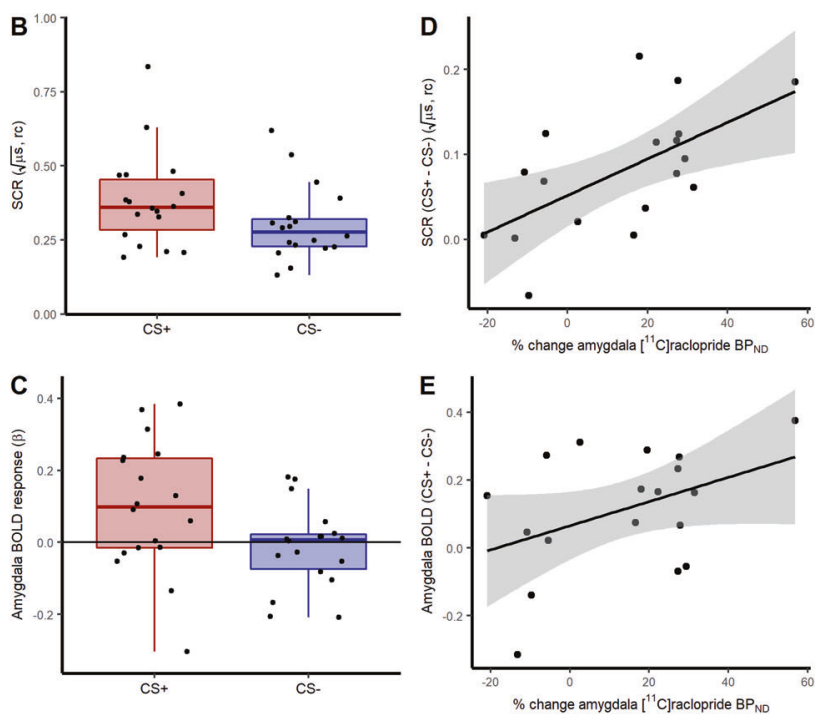

Fig. 4 Dopamine release in the amygdala during fear conditioning was co-localized with the neural memory trace and predicted both learning strength and memory trace activity. $A\left[{ }^{11} \mathrm{C}\right]$ raclopride binding potential $\left(\mathrm{BP}_{\mathrm{ND}}\right)$ was decreased during fear conditioning in a $320 \mathrm{~mm}{ }^{3}$ volume in the left $\left(Z=3.22 P_{\mathrm{FWE}}=0.017\right.$ family-wise error $[\mathrm{FWE}]$ corrected) and a $960 \mathrm{~mm}^{3}$ volume in the right amygdala $\left(Z=3.35 ; P_{\mathrm{FWE}}=0.012\right)$ with peak voxels at Montreal Neurological Institute (MNI) coordinates $-22,0,-14$ and $26,0,-14$ respectively, indicating dopamine release in these areas, and co-localized with blood-oxygenation-level dependent (BOLD) response to conditioned stimuli $(C S+-C S-)$ in the left $(Z=3.44$ $\left.P_{\mathrm{FWE}}=0.009 ; \mathrm{MNI}-22,0,-14\right)$ and right amygdala $\left(Z=3.04 ; P_{\mathrm{FWE}}=0.028 ; \mathrm{MNI}: 22,0,-14\right)$, indicating memory formation. Red clusters show dopamine release, green denotes clusters with greater neural activity to CS + than CS - shown here thresholded at $p<0.05$ for illustrative purposes, and yellow signifies overlap between dopamine release and learning-related neural activity within the amygdala. The fear conditioning procedure resulted in (B) discrimination between fear $(\mathrm{CS}+)$ and control $(\mathrm{CS}-)$ cues on skin conductance responses $(\mathrm{SCR})(\mathrm{t}(17)=$ $4.64, P=0.0001)$ and $(C)$ in an amygdala-located memory trace $(C S+>C S-)(t(17)=2.70, P=0.008)$. The amount of dopamine release in the amygdala predicted (D) learning strength $(r(16)=0.60, P=0.004,95 \% \mathrm{Cl}: 0.27-1)$ and $(\mathbf{E})$ amygdala memory trace activity $(r(16)=0.41, P=0.044$, $95 \% \mathrm{Cl}: 0.02-1)$. For the boxplots, the horizontal line indicates the median, the box the interquartile range (IQR), the whiskers the minimum of $1.5 \times$ IQR and minimum/maximum values, and the filled circles data for individual participants. For the scatterplots, shaded areas reflect standard error of means. rc: range corrected to each individual's maximum SCR. All statistical tests are one-sided tests of directed hypotheses.

inspection of the trial-by-trial SCR plot for each CS (Supplementary Fig. 4) point to initial gradual acquisition and later habituation. Blood-oxygenation-level dependent (BOLD) fMRI revealed higher $B O L D$ response to $C S+$ than $C S$ - in the amygdala (Fig. $4 \mathrm{~A}, \mathrm{C}$ ) that was linearly coupled to the skin conductance responses $(r(16)=$ $0.44, P=0.033,95 \% \mathrm{Cl}: 0.05-1.00)$, consistent with a vast literature underscoring amygdala as a key brain structure for aversive memory formation in humans and other animals [1].

\section{Dopaminergic facilitation of fear learning}

Next, we tested if dopamine release and learning strength are functionally coupled in the amygdala by correlating percent change in $\left[{ }^{11} \mathrm{C}\right]$ raclopride $\mathrm{BP}_{\mathrm{ND}}$ with SCR difference scores (CS+-CS-) and found a positive linear relationship $(r(16)=0.60, P=0.004,95 \% \mathrm{Cl}$ : $0.27-1$ ) (Fig. 4D). In contrast, the unconditioned response to electric shocks was not related to dopamine release in this region $(r(16)=$ $0.31, P=0.209,95 \% \mathrm{Cl}:-0.18$ to 0.68$)$. Percent change in amygdala $\left[{ }^{11} \mathrm{C}\right]$ raclopride $\mathrm{BP}_{\mathrm{ND}}$ also predicted learning-induced neural activity in the amygdala $(r(16)=0.41, P=0.044,95 \% \mathrm{Cl}$ : 0.02-1) (Fig. 4E). These findings confirm dose-response relations between amygdala dopamine release and learning strength, both in the peripheral and central nervous systems. Areas in the amygdala with endogenous dopamine release overlapped with the areas reflecting neural memory trace activity (Fig. 4A; Supplementary Fig. 5), demonstrating that dopamine release and neural activity were both functionally and anatomically coupled. Also, in the dorsal striatum, there was spatial overlap between dopamine release and neural activity $(\mathrm{CS}+>\mathrm{CS}-$ ) (Fig. 5A). However, we could not detect any relations between striatal dopamine release and striatal BOLD response (CS+-CS-) $(r(16)=$ $0.29, P=0.237,95 \% \mathrm{Cl}:-0.20$ to 0.67 ) (Fig. $5 \mathrm{~B}$ ) or SCR during conditioning $(r(16)=0.003, P=0.991,95 \% \mathrm{Cl}$ : -0.46 to 0.47$)$ (Fig. $5 \mathrm{C})$, nor between striatal BOLD response and SCR $(r(16)=0.27, P=0.271$,
95\% Cl: -0.22 to 0.66 ) (Fig. 5D). Follow-up analyses showed that SCR was more strongly associated to dopamine release in the amygdala than in the striatum $(Z=2.14, P=0.032)$, indicating specificity of dopaminergic facilitation of memory formation in the amygdala. No difference in dopamine-BOLD correlation coefficients between the amygdala and striatum could be detected $(Z=0.41, P=0.681)$.

\section{DISCUSSION}

We show that human fear conditioning is associated with endogenous dopamine release in the amygdala and that learning strength changes in concert with dopamine release in this brain region. This mirrors rodent studies demonstrating that fear conditioning is dependent on dopamine signaling in the amygdala [9-11, 13, 14, 36-38]. Using strict experimental controls, we confirm that fear conditioning induces peripheral and central nervous system learning. Statistically, we could further demonstrate that dopamine facilitates fear learning since learning strength was linked to dopamine release, while the strength of the unconditioned reaction was not. This is consistent with an interpretation that dopamine drives or is driven by learningrelated processes and suggesting specificity for learning-induced processes over stress reactivity. The association between dopamine and conditioned, but not unconditioned, responses is consistent with a study in rodents reporting that fear conditioning induced a higher rise in dopamine concentration than did shock presentations only [39]. Thus, our findings may also be interpreted in the light of dopamine release reflecting anticipation of salient stimuli. Two recent rodent studies have also showed that dopamine is released during foot shock and necessary for fear conditioning [38], and that the same dopaminergic neurons projecting from the ventral tegmental area to the basal amygdala 
A
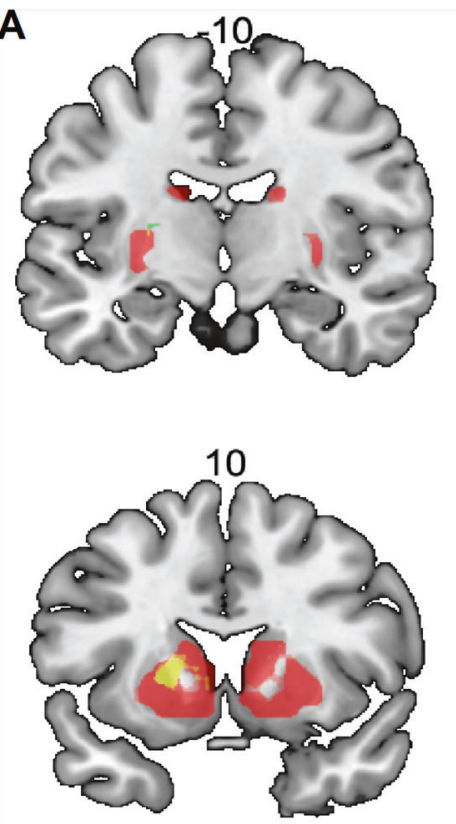

Dopamine release

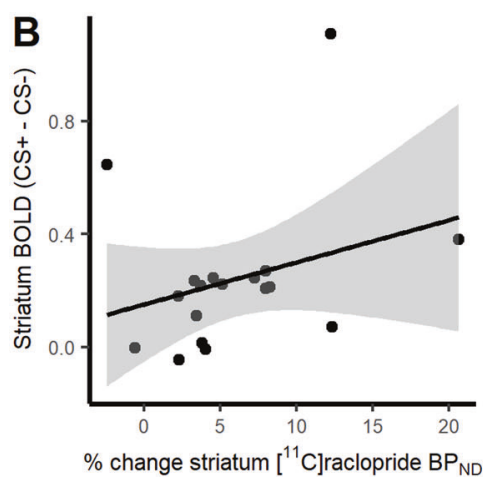

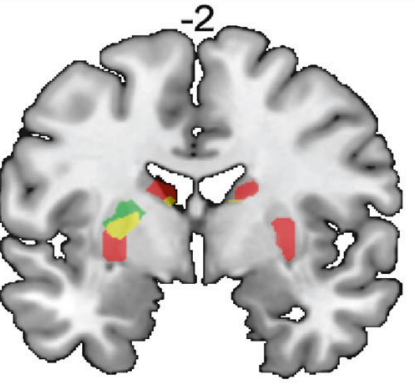
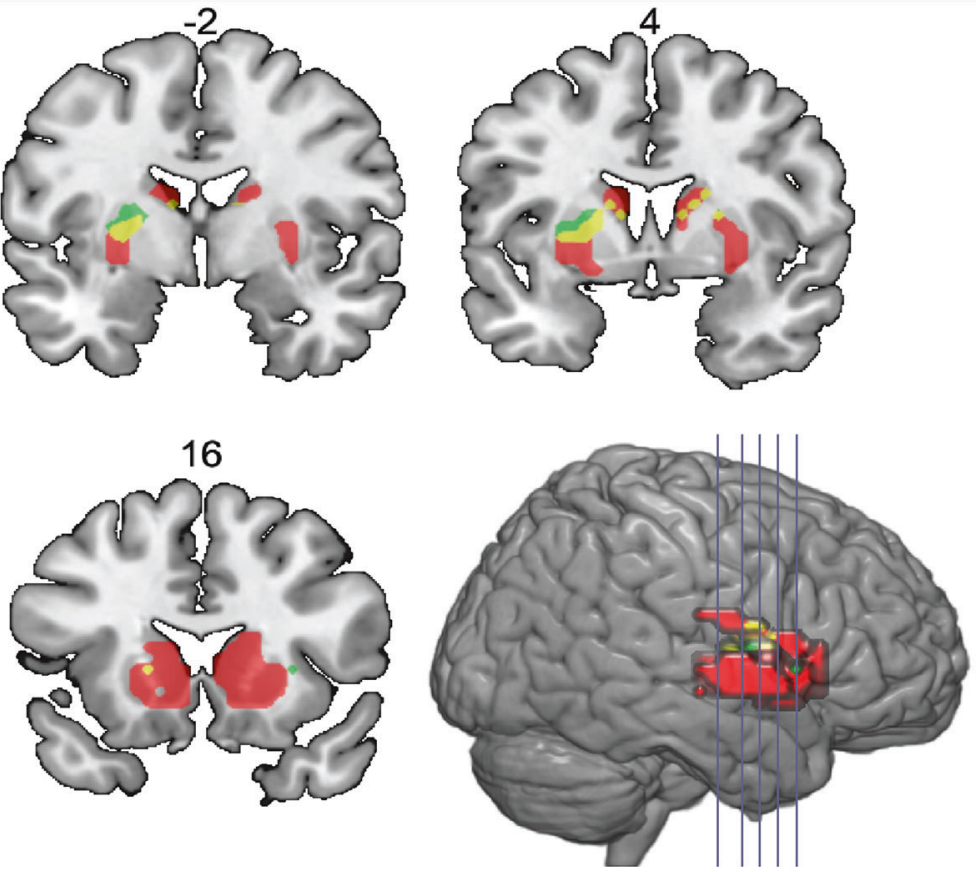

BOLD (CS+ > CS-)

Dopamine BOLD overlap

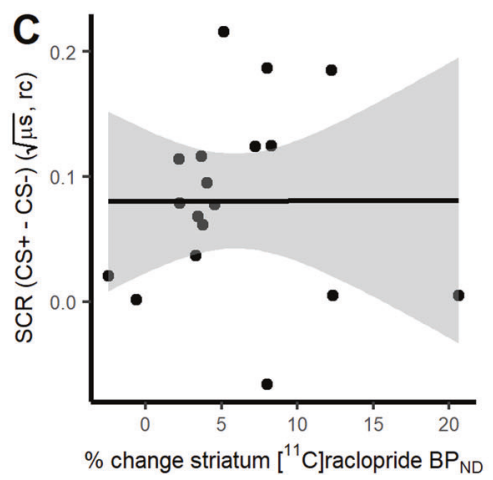

Fig. 5 Striatal dopamine release, neural activity and skin conductance response during fear conditioning. A $\left[{ }^{11} \mathrm{C}\right.$ ]raclopride binding potential $\left(\mathrm{BP}_{\mathrm{ND}}\right)$ was decreased during fear conditioning in a $7488 \mathrm{~mm}^{3}$ volume in the left $\left(Z=6.24 ; P_{\mathrm{FWE}}<.001\right.$ family-wise error [FWE] corrected) and a $8320 \mathrm{~mm}^{3}$ volume in the right striatum $\left(Z=6.54 ; P_{\mathrm{FWE}}<.001\right)$ with peak voxels at Montreal Neurological Institute coordinates $-18,8,-2$ and $22,8,-2$ respectively, indicating dopamine release in these areas, and co-localized with blood-oxygenation-level dependent (BOLD) response to conditioned stimuli $(C S+-C S-)$ in the left $\left(Z=4.87 ; P_{\mathrm{FWE}}=0.0002 ; \mathrm{MNI}-22,0,6\right)$ and right dorsal striatum $(Z=4.07$; $P_{\mathrm{FWE}}=0.008$; MNI: 22, 0, 10), indicating memory formation. Red clusters show dopamine release, green denotes clusters with greater neural activity to CS + than CS-, and yellow signifies overlap between dopamine release and learning-related neural activity within the amygdala. We could not detect any relation between percent change in striatal $\left[{ }^{11} \mathrm{C}\right]$ raclopride $\mathrm{BP}_{\mathrm{ND}}$, i.e., dopamine release, and (B) blood-oxygenationlevel dependent (BOLD) response to conditioned stimuli (CS+-CS-) $(r(16)=0.29, P=0.237,95 \% \mathrm{Cl}$ : -0.20 to 0.67$)$, or $(\mathbf{C})$ the peripheral measure of fear learning, skin conductance responses $(\mathrm{SCR})(r(16)=0.003, P=0.991,95 \% \mathrm{Cl}:-0.46$ to 0.47$)$. D Nor could we detect a relation between striatal BOLD response and SCR $(r(16)=0.27, P=0.271,95 \% \mathrm{Cl}:-0.22$ to 0.66$]$. rc range corrected to each individual's maximum SCR. For the scatter plots, shaded areas reflect standard error of means.

are activated by aversive and appetitive stimuli as well as cues that predict the aversive and appetitive stimuli [40]. These findings would rather point to the role of dopamine signaling salience. Indeed, dopamine has many different functions which act at different time-scales [41]. The PET measure of dopamine release used here does not have the temporal resolution to disentangle these time-varying effects.

One possible mechanism explaining our results is that dopamine facilitates long-term potentiation (LTP). LTP in the amygdala can cause fear learning [42], and because dopamine release facilitates LTP [43], our data are consistent with the notion that fear learning in humans is facilitated by dopamineinduced LTP and also with a recent rodent study demonstrating that D2 receptor stimulation facilitates fear learning [44]. We suggest that dopamine serves as a neurochemical guide to strengthen aversive memory formation and behavioral output.

Previous studies have attempted to identify characteristics that explain varying proneness for fear acquisition across individuals. Here, we contribute to this literature by showing that individual differences in dopamine release is related to strength of fear memory formation. This is in line with our previous findings of genetic contributions of the dopaminergic system (i.e., dopamine receptor D4 exon III variant) to fear acquisition [19].

Furthermore, there was evidence of an overlap between dopamine release in the striatum and learning-related activity. The voxel-wise analyses indicated overlap between PET and fMRI 
measures in the dorsal striatum, but the functional implications of this overlap is unclear as we could not detect any correlation between the two, or between these measures and the peripheral learning index. We can only speculate that the striatal dopamine release may be related to avoidance action programs.

Limitations of the current study deserve mentioning. First, the use of $\left[{ }^{11} \mathrm{C}\right]$ raclopride to measure dopamine release outside the striatum has been questioned. As mentioned in the introduction, this is based on the lack of change in BP in pharmacological occupancy studies. However, these studies have rarely included the amygdala or applied single scan bolus/infusion paradigms which arguably adds to the sensitivity of the measure. Moreover, test-retest reliability of amygdala $\left[{ }^{11} \mathrm{C}\right]$ raclopride $\mathrm{BP}$ has been reported to be high [21]. Also, here we found similar changes in $\left[{ }^{11} \mathrm{C}\right]$ raclopride $\mathrm{BP}_{\mathrm{ND}}$ in the amygdala and the striatum, although amygdala release had higher variability, whereas our control region, the frontal cortex did not indicate dopamine release. In addition, simulations (Supplementary Figs. 1 and 2) indicated that reduction in $\left[{ }^{11} \mathrm{C}\right]$ raclopride binding potential can be robustly measured in the amygdala using the nested two-step simplified reference tissue model (SRTM) approach used in this study. A principal shortcoming of the nested SRTM method used here is that it is insensitive to transient changes in tracer binding, and thus only detects prolonged changes. In situations with transient changes, other methods such as Ipnt-PET may be superior. Importantly, this does not affect the current results as the data showed prolonged reduction in tracer binding.

Using simultaneous measures of dopamine release and neural activity in a combined PET/MRI scanner we show that dopamine release during fear conditioning is linked to aversive memory formation. This is in line with animal studies showing that dopamine is critical for fear learning [13] and suggests that blocking dopaminergic signaling would reduce fear memory acquisition and that augmenting dopamine transmission during memory extinction would strengthen safety memory formation. This has clinical implications as manipulating fear and safety memories through extinction-based exposure forms the basis for cognitive behavioral therapy in anxiety and post-traumatic stress disorders [45]. Consistently, dopamine infusions in the rodent basolateral amygdala facilitate memory consolidation [46] and in humans, systemic L-DOPA administration strengthens safety memories during experimental fear extinction [47]. Dopamine facilitates memory formation, not only in the neural fear circuitry, but also in non-associative learning [48], instrumental conditioning [5], and in cognitive episodic memory [49]. Thus, we argue that dopamine represents an evolutionary conserved neurochemical mechanism supporting learning across multiple memory systems.

\section{REFERENCES}

1. LeDoux JE. Emotion circuits in the brain. Annu Rev Neurosci. 2000;23:155-84.

2. Agren T, Engman J, Frick A, Björkstrand J, Larsson E-M, Furmark T, et al. Disruption of reconsolidation erases a fear memory trace in the human amygdala. Science. 2012;337:1550-2.

3. Johansen JP, Cain CK, Ostroff LE, LeDoux JE. Molecular mechanisms of fear learning and memory. Cell. 2011;147:509-24.

4. LaBar KS, Gatenby JC, Gore JC, LeDoux JE, Phelps EA. Human amygdala activation during conditioned fear acquisition and extinction: a mixed-trial fMRI study. Neuron. 1998;20:937-45.

5. Wise RA. Dopamine, learning and motivation. Nat Rev Neurosci. 2004;5:483-94.

6. Murty VP, Ritchey M, Adcock RA, LaBar KS. fMRI studies of successful emotional memory encoding: a quantitative meta-analysis. Neuropsychologia. 2010;48:3459-69.

7. de la Mora MP, Gallegos-Cari A, Arizmendi-García Y, Marcellino D, Fuxe K. Role of dopamine receptor mechanisms in the amygdaloid modulation of fear and anxiety: Structural and functional analysis. Prog Neurobiol. 2010;90:198-216.

8. Jo YS, Heymann G, Zweifel LS. Dopamine neurons reflect the uncertainty in fear generalization. Neuron. 2018;100:916-25.e3.

9. Guarraci FA, Frohardt RJ, Kapp BS. Amygdaloid D1 dopamine receptor involvement in Pavlovian fear conditioning. Brain Res. 1999;827:28-40.
10. Guarraci FA, Frohardt RJ, Falls WA, Kapp BS. The effects of intra-amygdaloid infusions of a $D_{2}$ dopamine receptor antagonist on Pavlovian fear conditioning. Behav Neurosci. 2000;114:647-51.

11. Greba Q, Gifkins A, Kokkinidis L. Inhibition of amygdaloid dopamine D2 receptors impairs emotional learning measured with fear-potentiated startle. Brain Res. 2001;899:218-26.

12. Kim JJ, Jung MW. Neural circuits and mechanisms involved in Pavlovian fear conditioning: a critical review. Neurosci Biobehav Rev. 2006;30:188-202.

13. Fadok JP, Dickerson TMK, Palmiter RD. Dopamine is necessary for Cue-dependent fear conditioning. J Neurosci. 2009;29:11089-97.

14. Tsai H-C, Zhang F, Adamantidis A, Stuber GD, Bonci A, Lecea Lde, et al. Phasic firing in dopaminergic neurons is sufficient for behavioral conditioning. Science. 2009:324:1080-4.

15. Bäckman L, Nyberg L, Soveri A, Johansson J, Andersson M, Dahlin E, et al. Effects of working-memory training on striatal dopamine release. Science. 2011;333:718-718.

16. Aalto $S$, Brück $A$, Laine $M$, Någren $K$, Rinne JO. Frontal and temporal dopamine release during working memory and attention tasks in healthy humans: a positron emission tomography study using the high-affinity dopamine D2 receptor ligand [11C]FLB 457. J Neurosci. 2005;25:2471-7.

17. Badgaiyan RD, Fischman AJ, Alpert NM. Striatal dopamine release in sequential learning. Neurolmage. 2007:38:549-56.

18. Tessitore A, Hariri AR, Fera F, Smith WG, Chase TN, Hyde TM, et al. Dopamine modulates the response of the human amygdala: a study in Parkinson's disease. J Neurosci. 2002;22:9099-103.

19. Garpenstrand H, Annas P, Ekblom J, Oreland L, Fredrikson M. Human fear conditioning is related to dopaminergic and serotonergic biological markers. Behav Neurosci. 2001;115:358-64.

20. Uhlén $M$, Fagerberg L, Hallström BM, Lindskog C, Oksvold $P$, Mardinoglu A, et al. Tissue-based map of the human proteome. Science. 2015;347:1260419.

21. Karalija N, Jonassson L, Johansson J, Papenberg G, Salami A, Andersson M, et al. High long-term test-retest reliability for extrastriatal $11 \mathrm{C}$-raclopride binding in healthy older adults. J Cereb Blood Flow Metab. 2020;40:1859-68.

22. Farde L, Hall H, Ehrin E, Sedvall G. Quantitative analysis of D2 dopamine receptor binding in the living human brain by PET. Science. 1986;231:258-61.

23. Hall H, Farde L, Halldin C, Hurd YL, Pauli S, Sedvall G. Autoradiographic localization of extrastriatal D2-dopamine receptors in the human brain using [125I] epidepride. Synapse. 1996;23:115-23.

24. Lonsdorf TB, Menz MM, Andreatta M, Fullana MA, Golkar A, Haaker J, et al. Don't fear 'fear conditioning': methodological considerations for the design and analysis of studies on human fear acquisition, extinction, and return of fear. Neurosci Biobehav Rev. 2017;77:247-85.

25. Benedek M, Kaernbach C. A continuous measure of phasic electrodermal activity. J Neurosci Methods. 2010;190:80-91.

26. Boucsein W. Electrodermal activity. New York, NY: Plenum; 1992.

27. Boucsein W, Fowles DC, Grimnes S, Ben-Shakhar G, Roth WT, Dawson ME, et al. Publication recommendations for electrodermal measurements. Psychophysiology. 2012;49:1017-34.

28. Svarer C, Madsen K, Hasselbalch SG, Pinborg LH, Haugbøl S, Frøkjaer VG, et al. MR-based automatic delineation of volumes of interest in human brain PET images using probability maps. Neuroimage. 2005;24:969-79.

29. Lammertsma AA, Hume SP. Simplified reference tissue model for PET receptor studies. Neurolmage. 1996;4:153-8.

30. Gunn RN, Lammertsma AA, Hume SP, Cunningham VJ. Parametric imaging of ligand-receptor binding in PET using a simplified reference region model. Neurolmage. 1997;6:279-87.

31. Normandin MD, Schiffer WK, Morris ED. A linear model for estimation of neurotransmitter response profiles from dynamic PET data. Neurolmage. 2012;59:2689-99.

32. Yokoyama M, Suzuki E, Sato T, Maruta S, Watanabe S, Miyaoka H. Amygdalic levels of dopamine and serotonin rise upon exposure to conditioned fear stress without elevation of glutamate. Neurosci Lett. 2005;379:37-41.

33. Maldjian JA, Laurienti PJ, Kraft RA, Burdette JH. An automated method for neuroanatomic and cytoarchitectonic atlas-based interrogation of fMRI data sets. Neuroimage. 2003;19:1233-9.

34. Achterberg $M$, van der Meulen $M$. Genetic and environmental influences on MRI scan quantity and quality. Developmental Cogn Neurosci. 2019;38:100667.

35. R Core Team. R: a language and environment for statistical computing. Vienna, Austria: R Foundation for Statistical Computing; 2020.

36. Likhtik $E$, Johansen JP. Neuromodulation in circuits of aversive emotional learning. Nat Neurosci. 2019;22:1586-97.

37. Marowsky A, Yanagawa Y, Obata K, Vogt KE. A specialized subclass of interneurons mediates dopaminergic facilitation of amygdala function. Neuron. 2005;48:1025-37. 
38. Tang W, Kochubey O, Kintscher M, Schneggenburger RA. VTA to basal amygdala dopamine projection contributes to signal salient somatosensory events during fear learning. J Neurosci. 2020;40:3969-80.

39. Young AMJ, Joseph MH, Gray JA. Latent inhibition of conditioned dopamine release in rat nucleus accumbens. Neuroscience. 1993;54:5-9.

40. Lutas A, Kucukdereli H, Alturkistani O, Carty C, Sugden AU, Fernando K, et al. State-specific gating of salient cues by midbrain dopaminergic input to basal amygdala. Nat Neurosci. 2019;22:1820-33.

41. Schultz W. Neuronal reward and decision signals: from theories to data. Physiological Rev. 2015;95:853-951.

42. Rogan MT, Stäubli UV, LeDoux JE. Fear conditioning induces associative longterm potentiation in the amygdala. Nature. 1997;390:604-7.

43. Rossato JI, Bevilaqua LRM, Izquierdo I, Medina JH, Cammarota M. Dopamine controls persistence of long-term memory storage. Science. 2009;325:1017-20.

44. De Bundel D, Zussy C, Espallergues J, Gerfen CR, Girault J-A, Valjent E. Dopamine D2 receptors gate generalization of conditioned threat responses through mTORC1 signaling in the extended amygdala. Mol Psychiatry. 2016;21:1545-53.

45. Lange I, Goossens L, Michielse S, Bakker J, Vervliet B, Marcelis M, et al. Neural responses during extinction learning predict exposure therapy outcome in phobia: results from a randomized-controlled trial. Neuropsychopharmacol. 2020;45:534-41.

46. LaLumiere RT, Nguyen LT, McGaugh JL. Post-training intrabasolateral amygdala infusions of dopamine modulate consolidation of inhibitory avoidance memory: involvement of noradrenergic and cholinergic systems. Eur J Neurosci. 2004:20:2804-10.

47. Gerlicher AMV, Tüscher O, Kalisch R. L-DOPA improves extinction memory retrieval after successful fear extinction. Psychopharmacology. 2019;236:3401-12.

48. Ardiel EL, Giles AC, Yu AJ, Lindsay TH, Lockery SR, Rankin CH. Dopamine receptor DOP-4 modulates habituation to repetitive photoactivation of a $\mathrm{C}$. elegans polymodal nociceptor. Learn Mem. 2016;23:495-503.

49. Nyberg L, Karalija N, Salami A, Andersson M, Wåhlin A, Kaboovand N, et al Dopamine D2 receptor availability is linked to hippocampal-caudate functional connectivity and episodic memory. PNAS. 2016;113:7918-23.

\section{ACKNOWLEDGEMENTS}

We thank the staff at the Uppsala PET/MR facility and Uppsala PET Center for invaluable help with data collection. This work was supported by the Swedish Research Council, the Swedish Brain Foundation, the Swedish Society for Medical Research, the Kjell and Märta Beijer Foundation, Riksbankens Jubileumsfond - the Swedish Foundation for Humanities and Social Sciences, and Heumanska stiftelsen.

\section{AUTHOR CONTRIBUTIONS}

AF: Methodology, Formal analysis, Investigation, Data curation, Writing - Review \& Editing, Visualization, Project administration, Funding acquisition; JB: Methodology, Investigation, Writing - Review \& Editing; ML: Methodology, Software, Data curation, Supervision, Writing - Review \& Editing; AE: Investigation, Writing - Review \& Editing; MF: Conceptualization, Methodology, Writing - Original Draft, Supervision, Funding acquisition; FÅ: Conceptualization, Methodology, Writing - Review \& Editing, Funding acquisition.

\section{COMPETING INTERESTS}

The authors declare no competing interests.

\section{ADDITIONAL INFORMATION}

Supplementary information The online version contains supplementary material available at https://doi.org/10.1038/s41380-021-01400-x.

Correspondence and requests for materials should be addressed to Andreas Frick.

Reprints and permission information is available at http://www.nature.com/ reprints

Publisher's note Springer Nature remains neutral with regard to jurisdictional claims in published maps and institutional affiliations.

Open Access This article is licensed under a Creative Commons Attribution 4.0 International License, which permits use, sharing adaptation, distribution and reproduction in any medium or format, as long as you give appropriate credit to the original author(s) and the source, provide a link to the Creative Commons license, and indicate if changes were made. The images or other third party material in this article are included in the article's Creative Commons license, unless indicated otherwise in a credit line to the material. If material is not included in the article's Creative Commons license and your intended use is not permitted by statutory regulation or exceeds the permitted use, you will need to obtain permission directly from the copyright holder. To view a copy of this license, visit http://creativecommons. org/licenses/by/4.0/.

(c) The Author(s) 2021 